\title{
Effectiveness of two different HDR brachytherapy regimens with the same BED value in cervical cancer
}

\author{
Kamlesh Passi, BSc, Dip. RP', Than S Kehwar, PhD, DSc², Meenakshi Mittal, MD', Bikramjit Singh, MSc, Dip. RP' \\ Rajesh Vashistha, MD', Sureshchandra J Gupta, PhD³, JV Yakhmi, PhD ${ }^{4}$ \\ 'Department of Radiotherapy, M.D. Oswal Cancer Institute \& Research Foundation, Ludhiana (Pb), India, ${ }^{2}$ Medical Physics Division, Department \\ of Radiation Oncology. University of Pittsburgh Cancer Institute, UPMC Cancer Centers, Pittsburgh, PA, USA, ${ }^{3}$ Department of Physics, University of \\ Mumbai (MH), India, ${ }^{4}$ Physics Division, Bhabha Atomic Research Centre, Mumbai (MH), India
}

\begin{abstract}
Purpose: To analyze the effectiveness of biologically effective dose (BED) in two different regimens of HDR brachytherapy keeping the same total BED to point A and to compare the relationship of overall treatment time in terms of local control and bladder and rectal complications.

Material and methods: The study included two groups comprising a total of 90 cervical cancer patients who underwent external beam radiotherapy (EBRT) followed by HDR intracavitary brachytherapy (ICBT). EBRT treatment was delivered by a Co-60 teletherapy unit to a prescribed dose of 45 Gy with 1.8 Gy per fraction in 25 fractions over a period of five weeks. Parallel opposed anterior-posterior (AP/PA) fields with no central shielding were used, followed by the HDR ICBT dose, to point A, of either two fractions of $9.5 \mathrm{~Gy}$ with a gap of 10 days, or three fractions of $7.5 \mathrm{~Gy}$ with a gap of 7 days between the fractions. Gemcitabine (dose of $150 \mathrm{mg} / \mathrm{m}^{2}$ ) was given weekly to all the patients as a radiosensitizer. The calculate $\mathrm{BED}_{3}$ to point $\mathrm{A}$ was almost the same in both groups to keep the same late complication rates. The doses, and $\mathrm{BED}_{10}$ and $\mathrm{BED}_{3}$, were calculated at different bladder and rectal point as well as at the lymphatic trapezoid points. During and after treatment patients were evaluated for local control and complications for 24 months.

Results and Conclusions: Doses and BEDs at different bladder, rectal and lymphatic trapezoid points, local control, and complications in both HDR ICBT groups did not have statistically significant differences $(p>0.05)$. Both HDR ICBT schedules are well tolerable and equally effective.
\end{abstract}

Key words: cervical cancer, HDR brachytherapy, LQ model, ICRU-38.

\section{Purpose}

Cervical cancer is the second most common cancer among women in the world after breast cancer and is the most common cancer among Indian women [1-4]. A number of treatment modalities are being used for the treatment of the disease. Radiotherapy (RT) is one of them and plays an important role in the treatment of cervical cancer, where a combination of megavoltage external beam radiotherapy (EBRT) and intracavitary brachytherapy (ICBT) is an accepted definitive mode of treatment. The curative potential of RT in the management of cervical cancer is enhanced by the use of ICBT, which delivers a high radiation dose directly to the tumor while sparing (to an extent) the surrounding normal tissues. High-doserate (HDR) intracavitary brachytherapy for cervical cancer is widely used because of its advantages of a short treatment time, rigid immobilization, patient convenience, and out-patient procedure. Although a large number of fractionation schedules are in use for HDR brachytherapy, the optimal schedule has yet to be decided. Fractionation and dose adjustments of the total dose are crucial factors in lowering the frequency of complications without compromising the treatment results. The American Brachytherapy Society (ABS) has issued guidelines specifically for the use of HDR brachytherapy for cervical carcinoma [5]. They recommended that the individual fraction size should be $7.5 \mathrm{~Gy}$ and the range of fractions should be 4 to 8 , depending on the fraction size, and have also included a caution that "these recommendations are intended as guidelines and the suggested fractionation schemes have not been thoroughly tested" [5]. Hence, thorough clinical testing of EBRT in combination with HDR brachytherapy has become an important component in radiotherapy management of patients with cervical cancer. On the other hand, the International Commission on Radiation Units and Measurements (ICRU) published its report no. 38 on Dose and volume specifications for reporting intracavitary therapy in gynecology and recommended reference points for the rectum and bladder along with some other important ones [6]. Many authors have studied 
the impact of dose variation on different points in the rectum and bladder along with ICRU points [6-8]. Many times planned treatment may change from the original due to unavoidable circumstances. These variations during treatment should be unified by radiobiological parameters such as biologically effective dose (BED). Mandal et al. [9] have done a retrospective study to analyze the predictive utility of radiobiological parameters in treatment $\mathrm{BED}_{2.5}$ for late reacting tissues and $\mathrm{BED}_{10}$ for acute reacting tissues in relation to overall treatment time using a low dose rate (LDR) system. For over four decades numerous studies have demonstrated its effectiveness and safety, and HDR is now accepted as an equivalent alternative to LDR brachytherapy [10-16]. As different treatment regimens are used at various centers, radiobiological equivalency has an important role to help clinicians in evaluating various treatment schedules quantitatively to predict the treatment outcome [17-19].

Radiobiological comparisons have also been done for different regimens of ICBT using the LDR dose delivery mode where it was found that more than one fraction did not improve the cure rate, nor did it reduce the complication rate [20]. In this prospective study, two groups of patients with cervical cancer who received a combination of EBRT and HDR ICBT were analyzed to assess the local control of disease and complication rate. In both groups of patients, the EBRT part of the treatment was the same, while HDR ICBT was varied, but the total planned BED at $\alpha / \beta=3$ Gy and overall treatment time were kept nearly the same (within $\pm 0.5 \%$ ), while BED at $\alpha / \beta=10$ Gy was within $\pm 3.0 \%$ without compromising tumor control.

\section{Material and methods}

Ninety cervical cancer patients treated between September 2006 and February 2008 were included in this study. Out of these, 16 patients were defaulted after EBRT, while 4 patients left after the first ICBT application. Only 70 patients were available for radiobiological evaluation. All the patients received EBRT to a prescribed dose of 45 Gy with 1.8 Gy per fraction in 25 fractions over a period of 5 weeks with 5 fractions per week by a Co-60 teletherapy unit. No central shielding was used in these patients. Weekly gemcitabine (dose of $150 \mathrm{mg} / \mathrm{m}^{2}$ ) was given to all the patients as a radiosensitizer. In the HDR ICBT appli-

Table 1. Distribution of patients as per FIGO staging, histopathology, and age

\begin{tabular}{lll} 
Patient characteristics & Group I & Group II \\
\hline Figo staging & & \\
\hline Stage II & $22 / 32(68.7 \%)$ & $26 / 38(68.4 \%)$ \\
\hline Stage III & $10 / 32(31.3 \%)$ & $12 / 38(31.6 \%)$ \\
\hline Histopathology & & \\
\hline Squamous cell carcinoma & 32 & 36 \\
\hline Adenocarcinoma & 0 & 2 \\
\hline Mean age (years) & 51.97 & 52.54 \\
\hline Age range (years) & $30-73$ & $39-78$
\end{tabular}

cation the dose was prescribed to point $A$, where point $A$ is defined as $2 \mathrm{~cm}$ lateral perpendicular to the midline of the intrauterine canal and $2 \mathrm{~cm}$ cephalad along the tandem from the external cervical os. For the HDR ICBT applications, patients were divided into two groups. In Group I patients received two fractions of 9.5 Gy each separated by 10 days, while in Group II patients received three fractions of 7.5 Gy each separated by 7 days. Patients in Group I received a total dose of 64 Gy (45 Gy by EBRT and 19 Gy by HDR brachytherapy with 9.5 Gy in 2 fractions), while in Group II total dose was 67 Gy (45 Gy by EBRT and 22.5 Gy by HDR brachytherapy with 7.5 Gy in 3 fractions) to point $\mathrm{A}$. The $\mathrm{BED}_{3}$ to point $\mathrm{A}$ was kept almost the same in both the groups, calculated using a linear quadratic (LQ) model.

BED is the cumulative biologically effective dose and may be expressed as

$$
\begin{aligned}
\mathrm{BED}=\mathrm{BED}_{\mathrm{EBRT}}+\mathrm{BED}_{\text {ICBT }} \\
=\text { nd }[1+\{\mathrm{d} /(\alpha / \beta)\}]+\sum_{i=1}^{N} \mathrm{R}_{\mathrm{i}}\left[1+\left\{\mathrm{R}_{\mathrm{i}}(\alpha / \beta)\right\}\right]
\end{aligned}
$$

Where $d$ is the EBRT dose per fraction delivered in $n$ number of fractions, and $R_{i}$ is the HDR ICBT dose of the $i^{\text {th }}$ fraction, where $N=1$ and 2 for Group I, and 1, 2 and 3 for Group II.

Corresponding total planned $\mathrm{BED}_{10}$ (without proliferation correction) and $\mathrm{BED}_{3}$, for EBRT plus HDR ICBT, at point A were 90.15 Gy and 151.17 Gy for Group I, and 92.47 Gy and 150.75 Gy for Group II, respectively. The total number of patients was 32 and 38 in Group I and II with mean age 51.97 years (range $30-73$ ) and 52.54 years (range 39-78), respectively. Mean treatment time in Group I and Group II was 75 days and 80 days, respectively. Patient characteristics are shown in Table 1.

During EBRT, complications related to skin, rectum, bladder and small bowel were recorded on a weekly basis and on every follow-up afterwards as per LENTSOMA specifications [21]. Patients were assessed for local response and normal tissue complications clinically, histopathologically and radiologically. Sigmoidoscopy/proctoscopy was also done in patients with rectal bleeding.

\section{Dose calculation and treatment planning}

Details of the dose calculations and treatment planning are described elsewhere [22-24]. In brief, orthogonal radiographs were taken for all the ICBT insertions individually. Radio-opaque rectal marker was inserted in the rectum for localization. Radio-opaque gauze packing was done to localize the anterior rectal wall. To visualize the bladder, 7 cc of contrast was filled in Foley's catheter balloon and radio-opaque gauze was packed to localize the posterior vaginal wall. Using orthogonal radiographs doses were computed on various points of interest using the Nucletron Plato Planning System ${ }^{\circledR}$. In the study, in addition to ICRU-38 rectal and bladder reference points, additional rectal points were also selected as two above the ICRU rectal points and two below at $1.0 \mathrm{~cm}$ apart, while in the bladder, four additional points were placed along with the ICRU reference point to mark five proximal sides of 
Table 2. Recurrence rate and follow-up of patients in Group I

\begin{tabular}{|c|c|c|c|c|c|}
\hline Response/Time period & 1 month & 3 months & 6 months & 12 months & 24 months \\
\hline Lost to follow-up & Nil & Nil & $1(3.1 \%)$ & $3(10.3 \%)$ & $1(3.8 \%)$ \\
\hline Recurrence/metastasis & Nil & Nil & $1(3.1 \%)$ & Nil & Nil \\
\hline Expired & Nil & Nil & $1(3.1 \%)$ & Nil & $1(3.8 \%)$ \\
\hline Patients evaluated & 32 & 32 & 32 & 29 & 26 \\
\hline
\end{tabular}

Table 3. Recurrence rate and follow-up of patients in Group II

\begin{tabular}{|c|c|c|c|c|c|}
\hline Response/Time period & 1 month & 3 months & 6 months & 12 months & 24 months \\
\hline Lost to follow-up & Nil & $1(2.6 \%)$ & $1(2.7 \%)$ & $1(2.9 \%)$ & Nil \\
\hline Recurrence/metastasis & Nil & Nil & $2(5.4 \%)$ & Nil & $2(5.9 \%)$ \\
\hline Expired & Nil & Nil & $1(2.7 \%)$ & Nil & $2(5.9 \%)$ \\
\hline Patients evaluated & 38 & 38 & 37 & 35 & 34 \\
\hline
\end{tabular}

the balloon. The reference dose prescription was given at point $A$ and doses were recorded for points $A, A^{\prime}, B, B '$ along with five bladder and five rectal points.

\section{Follow-up and evaluation}

After completion of EBRT and HDR ICBT, all patients were called for follow-up in the first year at monthly intervals, quarterly in the second year and half yearly in the third year. All patients were evaluated to estimate their response in terms of local control and normal tissue complications for small bowel, bladder, and rectum at $1,3,6,12$, and 24 month intervals.

\section{Statistical analysis}

The statistical endpoints for this study were the KaplanMeier estimates of the local control and normal tissue complications evaluated from the date of completion of the irradiation. Student's t-test was used to analyze the differences in the results of Group I and Group II patients and also in doses and BEDs at different points and applications of HDR ICBT.

\section{Results}

Figures 1 and 2 show the plots of the Kaplan-Meier analysis of the complete response and partial response, respectively, for the period of 24 months. Tables 2 and 3 list the number lost to follow-up, recurrence/metastasis, patient death and number of patients evaluated at 1, 3, 6, 12, 24 months after completion of irradiation for both patient groups. At one month after completion of irradiation, in Group I patients, $25(78.1 \%)$ had a complete response and $7(21.9 \%)$ had a partial response, whereas in Group II patients, $31(81.6 \%)$ and $7(18.4 \%)$ had a complete and partial response, respectively. Three patients $(42.8 \%)$ who had a partial response in Group I at a 1 month interval had complete remission at 3 months, while only $1(14.3 \%)$ patient had a partial response in Group II. At 6 months, 2 patients expired in Group I, one due to cardiac disease and the second due to distant metastasis as ascites and pleural effusion and

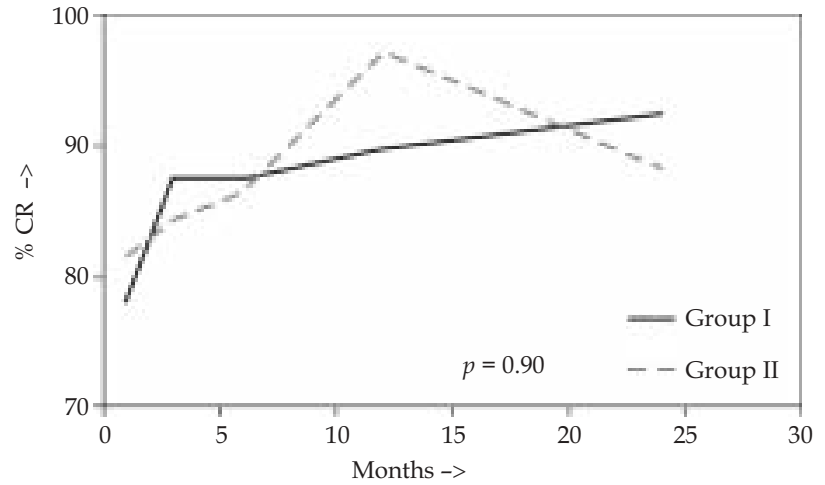

Fig. 1. Plots between follow-up time and complete tumor response estimated using Kaplan-Meier analysis in Groups I and II

1 patient was lost to follow-up. In Group II, only 1 patient died due to disease and 1 patient was lost to follow-up at a 6 month interval. In the study, at 24 months in Group I, only $26(81.25 \%)$ patients were evaluable, and 24 (92.3\%) had a complete response. One patient expired and 1 patient was lost to follow-up. In Group II, 34 (88.2\%) evaluable patients had a complete response, 1 developed distant metastasis to the lungs, and another 1 developed para-aortic lymph node metastasis. Both patients were given chemotherapy and expired. Two other patients expired due to other medical problems at 24 months post-treatment. Patients who died due to causes other than the cancer and lost to follow-up were censored from the study. The comparison of complete and partial responses, shown in Figs. 1 and 2, reveals that there were no statistically significant differences between the two groups (Student's t-test, $p>0.05$ ).

During and just after completion of irradiation, the majority of the toxicities encountered were related to the gastrointestinal system, Grade 1-2 nausea, vomiting and loss of appetite seen in almost all patients in both groups. Only 1-2 patients in each group encountered Grade 2-3 small bowel complications in the form of loose stools which required hospitalization and were medically managed 


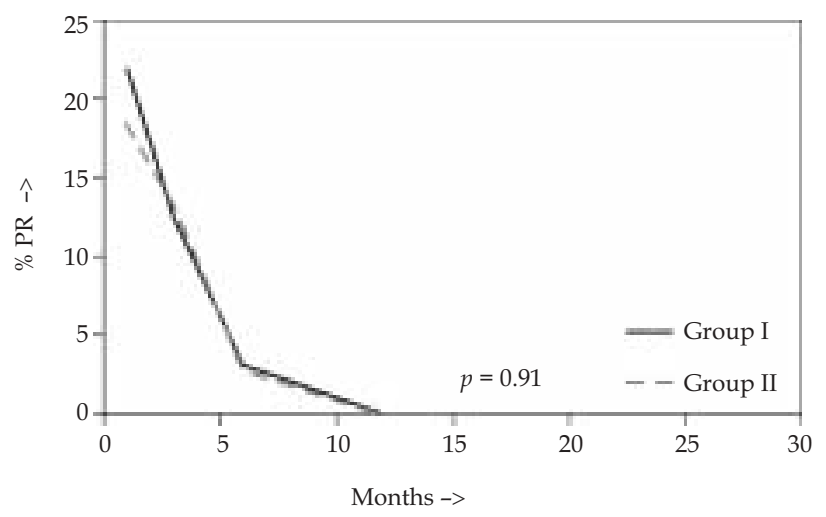

Fig. 2. Plots between follow-up time and partial tumor response estimated using Kaplan-Meier analysis in Groups I and II

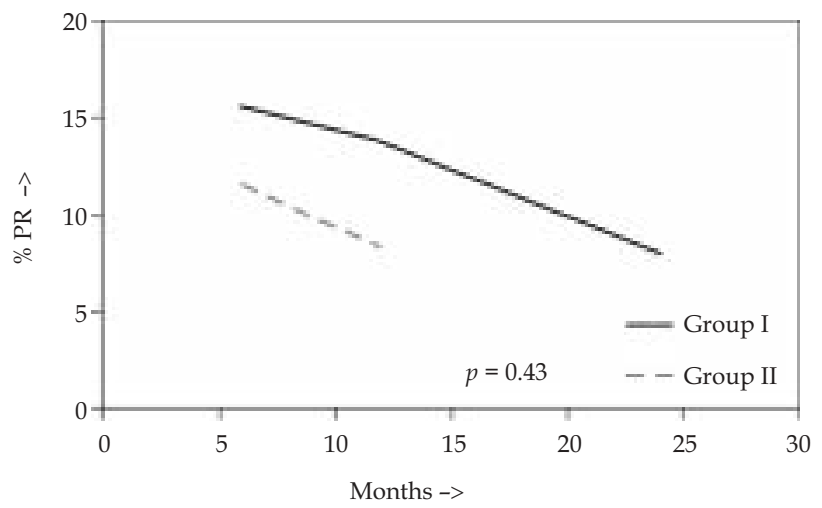

Fig. 3. Plots between follow-up time and Grade 2 plus Grade 3 rectal complications estimated by Kaplan-Meier analysis in Groups I and II

(1 patient in Group I and 2 patients in Group II). None of the patients had small bowel complications in the subsequent period and after 6 months from completion of irradiation. No patients reported any complications in the bladder, in both groups, during the period of evaluation. Figure 3 represents the Grade 2 plus Grade 3 rectal complications in Group I and Group II patients, and shows no statistically significant differences between the two groups (Student's t-test, $\mathrm{p}>0.05$ ). In Group I, late rectal toxicity (bleeding), Grade 2 and Grade 3, at 6 months was seen in 4 patients $(12.5 \%)$, and in 1 ( $3.1 \%)$ patient, respectively, whereas in Group II, after 12 months of followup, Grade 2 and Grade 3 toxicity was seen in 3 patients $(8.5 \%)$, and in $1(2.9 \%)$ patient, respectively. At 24 months of follow-up each group had 2 patients with Grade 2 rectal toxicity $(7.7 \%$ in Group I and $5.9 \%$ in Group II).

Doses delivered to different bladder, rectal, and lymphatic trapezoid points in both groups are listed in Table 4. Doses are reported as the mean of that received in HDR ICBT application with one standard deviation (mean dose $\pm 1 \mathrm{SD}$ ). For corresponding doses at different points in both groups BEDs were calculated for $\alpha / \beta=10$ Gy and 3 Gy. Results are presented in Tables 5 and 6, respectively.

\section{Discussion}

Chemoradiation is nowadays considered to be the standard treatment of choice in all stages of cervical cancer with a combination of EBRT and ICBT. HDR ICBT is now used worldwide in the management of patients with cervical cancer treated by radiation therapy. Several regimens have been studied, most of them with reasonable efficacy and toxicity profiles that are comparable to those of LDR brachytherapy regimens, but still the optimum fractionation schedule remains debatable and controversial.

The fractionation schedules used in this study were also empirically developed and the BED to point A was kept the same in both groups, calculated using the LQ model. At MD Oswal Cancer Institute, there was a shift from LDR to HDR in the year 2006. The HDR ICBT doses were calculated to obtain a BED equivalence of the total BED of EBRT and LDR ICBT doses. The HDR ICBT was started with a dose of $7.5 \mathrm{~Gy} /$ fraction delivered in 3 fractions. When patients showed good tolerance to this fractionation schedule, a treatment schedule of $9.5 \mathrm{~Gy} /$ fraction delivered in 2 fractions was used, keeping the same total BED to point $\mathrm{A}$ as it was for $7.5 \mathrm{~Gy} \times 3$ fractions with EBRT.

Many other authors have reported results using 2 or 3 fractions. In a randomized trial comparing HDR ICBT versus LDR ICBT, Patel et al. [14] studied 412 patients treated either by 18 Gy in 2 fractions of 9 Gy each with HDR ICBT or 35 Gy by continuous LDR ICBT, and both groups received 45 Gy EBRT. The survival rate and local control were not significantly different between the two groups. The complication rate was also comparable, with no evidence of increased toxicity in the HDR ICBT patients despite the small fractionation number. Wong et al. [16] treated 220 Chinese patients using either 3 or 4 fractions of HDR ICBT in combination with EBRT. The 5-year actuarial late complication rate (grade 3 or above) was $2.8 \%$, with no significant difference in complication rate between 3 or 4 fractions. In this study the 2-year late rectal complication rate was about $8 \%$, with no significant difference $(p>0.05)$ in complication rate between the two groups. $\mathrm{BED}_{10}$ or $\mathrm{BED}_{3}$ at none of the points, of both the groups, had statistically significant differences $(p>0.05)$. The patients whose BED10 was in the range of 59 Gy to 65 Gy developed small bowel complications of Grade 2-3 during or just after completion of irradiation in both the groups, but there were no statistically significant differences $(p>0.05)$. Similarly, the rectal complications and $\mathrm{BED}_{10}$ and $\mathrm{BED}_{3}$, in both the groups, did not show statistically significant differences $(p>0.05)$. Only those patients developed Grade 2 and Grade 3 late rectal complications who received a $\mathrm{BED}_{3}$ value of $120 \mathrm{~Gy}$ or higher to the rectal point irrespective of the place of the point. It is not necessary that this should be specifically at the ICRU reference point.

In another study by Sood et al. [25], 49 patients with stage I-III disease were treated with EBRT (45 Gy with or without parametrial boost) plus 2 fractions of 7-11 Gy each of HDR ICBT. The actuarial risk of Grade 2 or worse toxicity was 5\% and the overall 5 -year survival rate was $78 \%$. In another study, Han et al. [26] presented the results of 88 patients with cervical carcinoma treated with HDR brachytherapy using smaller fraction sizes (mean 3.8 Gy) with an increased 
Table 4. Doses from brachytherapy applications at different points of bladder, rectum and trapezoid in patients of both groups

\begin{tabular}{|c|c|c|c|c|c|}
\hline & \multicolumn{2}{|c|}{ Group I } & \multicolumn{3}{|c|}{ Group II } \\
\hline & $1^{\text {st IC }}$ & $2^{\text {nd }} \mathrm{IC}$ & $1^{\text {st }}$ IC & $2^{\text {nd }} \mathrm{IC}$ & $3^{\text {rd }}$ IC \\
\hline Bladder Pt. 1 (ICRU Pt.) & $4.67 \pm 1.90$ & $5.37 \pm 2.54$ & $3.39 \pm 1.24$ & $3.37 \pm 0.87$ & $3.49 \pm 0.95$ \\
\hline Bladder Pt. 2 & $3.68 \pm 1.13$ & $4.07 \pm 1.46$ & $2.76 \pm 0.86$ & $2.69 \pm 0.66$ & $2.75 \pm 0.70$ \\
\hline Bladder Pt. 3 & $4.45 \pm 1.69$ & $4.93 \pm 1.73$ & $2.88 \pm 0.90$ & $3.09 \pm 0.71$ & $3.27 \pm 0.84$ \\
\hline Bladder Pt. 4 & $2.60 \pm 1.29$ & $2.86 \pm 1.42$ & $1.73 \pm 0.75$ & $1.80 \pm 0.61$ & $2.21 \pm 0.50$ \\
\hline Bladder Pt. 5 & $4.49 \pm 1.62$ & $5.00 \pm 1.95$ & $3.03 \pm 1.01$ & $3.04 \pm 0.94$ & $3.31 \pm 1.26$ \\
\hline Rectal Pt. 1 (ICRU Pt.) & $5.07 \pm 1.96$ & $5.06 \pm 1.46$ & $3.56 \pm 1.16$ & $3.52 \pm 0.92$ & $3.77 \pm 0.90$ \\
\hline Rectal Pt. 3 & $4.73 \pm 1.98$ & $4.68 \pm 1.20$ & $3.47 \pm 1.24$ & $3.25 \pm 0.94$ & $3.58 \pm 0.90$ \\
\hline Rectal Pt. 4 & $4.50 \pm 1.91$ & $4.43 \pm 1.27$ & $3.18 \pm 0.98$ & $3.23 \pm 0.81$ & $3.39 \pm 0.79$ \\
\hline Rectal Pt. 5 & $3.31 \pm 0.78$ & $3.41 \pm 0.96$ & $2.49 \pm 0.64$ & $2.63 \pm 0.73$ & $2.70 \pm 0.69$ \\
\hline Rt. Para Aortic* & $0.35 \pm 0.16$ & $0.34 \pm 0.12$ & $0.33 \pm 0.15$ & $0.28 \pm 0.08$ & $0.30 \pm 0.11$ \\
\hline Lt. Para Aortic* & $0.37 \pm 0.16$ & $0.37 \pm 0.13$ & $0.34 \pm 0.15$ & $0.30 \pm 0.09$ & $0.34 \pm 0.13$ \\
\hline Rt. Comn. Iliac & $1.71 \pm 1.14$ & $1.44 \pm 0.88$ & $1.41 \pm 0.77$ & $1.20 \pm 0.40$ & $1.25 \pm 0.41$ \\
\hline Lt. Comn. Iliac & $1.42 \pm 0.74$ & $1.63 \pm 0.73$ & $1.34 \pm 0.52$ & $1.32 \pm 0.47$ & $1.34 \pm 0.50$ \\
\hline Rt. Ext. Iliac & $2.04 \pm 0.68$ & $1.84 \pm 0.48$ & $1.64 \pm 0.40$ & $1.54 \pm 0.31$ & $1.54 \pm 0.23$ \\
\hline
\end{tabular}


Table 5. BED 10 from EBRT plus ICBT plans at different points of bladder, rectum and trapezoid in patients of both groups

\begin{tabular}{|c|c|c|c|c|c|c|c|}
\hline & \multicolumn{3}{|c|}{ Group I } & \multicolumn{4}{|c|}{ Group II } \\
\hline & $1^{\text {st IC }}$ & $2^{\text {nd }}$ IC & Total BED $=$ EBRT $+\mathrm{IC}$ & $1^{\text {st IC }}$ & $2^{\text {nd }}$ IC & 3rd IC & Total BED $=$ EBRT $+\mathrm{IC}$ \\
\hline Points & $\mathrm{BED}_{10} \pm 1 \mathrm{SD}$ & $\mathrm{BED}_{10} \pm 1 \mathrm{SD}$ & $\mathrm{BED}_{10} \pm 1 \mathrm{SD}$ & $\mathrm{BED}_{10} \pm 1 \mathrm{SD}$ & $\mathrm{BED}_{10} \pm 1 \mathrm{SD}$ & $\mathrm{BED}_{10} \pm 1 \mathrm{SD}$ & $\mathrm{BED}_{10} \pm 1 \mathrm{SD}$ \\
\hline & (Gy) & (Gy) & (Gy) & (Gy) & (Gy) & (Gy) & (Gy) \\
\hline Bladder Pt. 1 (ICRU Pt.) & $7.20 \pm 3.96$ & $8.86 \pm 6.38$ & $69.16 \pm 8.11$ & $4.68 \pm 2.18$ & $4.59 \pm 1.50$ & $4.79 \pm 1.67$ & $67.16 \pm 4.54$ \\
\hline Bladder Pt. 2 & $5.15 \pm 1.99$ & $5.92 \pm 2.81$ & $64.17 \pm 4.06$ & $3.58 \pm 1.42$ & $3.45 \pm 1.02$ & $3.55 \pm 1.12$ & $63.68 \pm 2.60$ \\
\hline Bladder Pt. 3 & $6.70 \pm 3.35$ & $7.65 \pm 3.57$ & $67.45 \pm 6.13$ & $3.79 \pm 1.52$ & $4.08 \pm 1.17$ & $4.41 \pm 1.45$ & $65.38 \pm 3.48$ \\
\hline Bladder Pt. 4 & $3.43 \pm 2.23$ & $3.86 \pm 2.59$ & $60.39 \pm 4.66$ & $2.03 \pm 1.10$ & $2.12 \pm 0.91$ & $2.71 \pm 0.70$ & $56.86 \pm 2.28$ \\
\hline Bladder Pt. 5 & $6.75 \pm 3.16$ & $7.86 \pm 4.39$ & $67.71 \pm 6.06$ & $4.04 \pm 1.68$ & $4.05 \pm 1.56$ & $4.55 \pm 2.41$ & $65.74 \pm 4.99$ \\
\hline Rectal Pt. 1 (ICRU Pt.) & $7.99 \pm 4.19$ & $7.83 \pm 3.12$ & $68.92 \pm 5.63$ & $4.95 \pm 1.99$ & $4.84 \pm 1.55$ & $5.27 \pm 1.64$ & $68.16 \pm 4.23$ \\
\hline Rectal Pt. 2 & $6.08 \pm 3.34$ & $5.98 \pm 1.94$ & $65.15 \pm 4.41$ & $4.37 \pm 2.32$ & $3.84 \pm 1.63$ & $4.34 \pm 1.48$ & $65.65 \pm 4.83$ \\
\hline Rectal Pt. 3 & $7.35 \pm 4.02$ & $7.00 \pm 2.30$ & $67.44 \pm 5.16$ & $4.83 \pm 2.12$ & $4.39 \pm 1.57$ & $4.94 \pm 1.60$ & $67.26 \pm 4.28$ \\
\hline Rectal Pt. 4 & $6.87 \pm 4.29$ & $6.55 \pm 2.49$ & $66.52 \pm 5.73$ & $4.28 \pm 1.63$ & $4.33 \pm 1.36$ & $4.60 \pm 1.36$ & $66.31 \pm 3.50$ \\
\hline Rectal Pt. 5 & $4.47 \pm 1.27$ & $4.66 \pm 1.70$ & $62.23 \pm 2.20$ & $3.15 \pm 0.97$ & $3.37 \pm 1.15$ & $3.48 \pm 1.11$ & $63.10 \pm 2.25$ \\
\hline Rt. Para Aortic* & $0.37 \pm 0.17$ & $0.35 \pm 0.13$ & $0.72 \pm 0.29$ & $0.34 \pm 0.16$ & $0.28 \pm 0.09$ & $0.31 \pm 0.12$ & $0.94 \pm 0.35$ \\
\hline Lt. Para Aortic ${ }^{\star}$ & $0.39 \pm 0.18$ & $0.39 \pm 0.14$ & $0.77 \pm 0.30$ & $0.35 \pm 0.16$ & $0.31 \pm 0.10$ & $0.35 \pm 0.14$ & $1.01 \pm 0.37$ \\
\hline Rt. Comn. Iliac & $2.12 \pm 1.65$ & $1.72 \pm 1.29$ & $56.94 \pm 2.67$ & $1.67 \pm 1.11$ & $1.35 \pm 0.51$ & $1.42 \pm 0.52$ & $57.55 \pm 1.70$ \\
\hline Lt. Comn Iliac & $1.68 \pm 1.09$ & $1.94 \pm 1.06$ & $56.72 \pm 1.66$ & $1.55 \pm 0.70$ & $1.52 \pm 0.61$ & $1.54 \pm 0.66$ & $57.71 \pm 1.57$ \\
\hline Rt. Ext. Iliac & $2.51 \pm 1.01$ & $2.20 \pm 0.66$ & $57.80 \pm 1.37$ & $1.92 \pm 0.56$ & $1.79 \pm 0.42$ & $1.78 \pm 0.30$ & $58.58 \pm 0.77$ \\
\hline Lt. Ext. \|liac & $1.99 \pm 0.54$ & $2.27 \pm 0.61$ & $57.35 \pm 0.86$ & $1.64 \pm 0.35$ & $1.71 \pm 0.38$ & $1.67 \pm 0.27$ & $58.11 \pm 0.58$ \\
\hline
\end{tabular}


Table 6. $\mathrm{BED}_{3}$ from EBRT plus ICBT plans at different points of bladder, rectum and trapezoid in patients of both groups

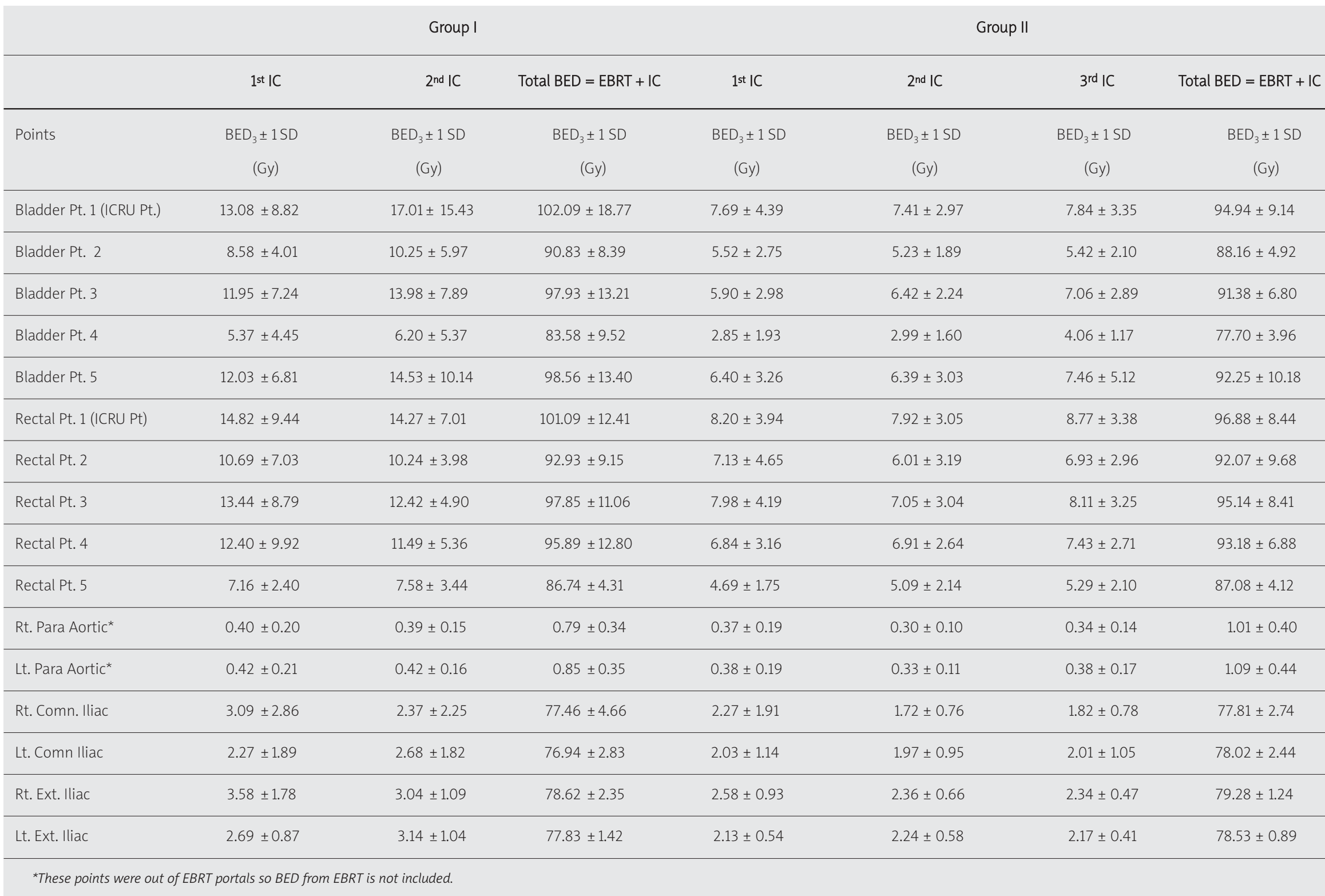

"The points were out of EBRT portals so BED from EBRT is not included. 
number of fractions ranging from 8 to 12 . The rate of grade 3-4 complications was 3.4\%. Patel et al. [27] reported 3.31\% grade 3 or more toxicity and the actuarial local control and 5 -year survival rate of $74.5 \%$ and $62.03 \%$, respectively, in a study of 113 patients with stage I-III disease treated with 9 Gy/fraction of 2-5 fractions of HDR ICBT.

In the present study, at 2 years of follow-up, we observed local control of $92.3 \%$ and $88.2 \%$ in Group I and Group II patients with the actuarial local control rate of $75 \%$ and $78.9 \%$ in Group I and Group II, respectively.

Souhami et al. [28] gave the long-term results of HDR brachytherapy in cervical cancer using a small number of fractions. At a median follow-up of 86.3 months for patients at risk, the 5, 10 and 15 year overall survival rates were $57 \%, 52 \%$ and $47 \%$, respectively. Overall gastrointestinal and genitourinary actuarial complication rates were $15 \%$ and $8 \%$, respectively. In their experience, HDR ICBT using 3 insertions was well tolerated and the results were comparable to HDR ICBT using a larger number of fractions and to low dose rate brachytherapy. The patients were treated with EBRT to the whole pelvis (median dose $45 \mathrm{~Gy}$ ) and HDR ICBT (median dose of 24 Gy at point A) in 3 insertions given weekly.

\section{Conclusions}

The initial opinion was that HDR brachytherapy given in smaller doses per fraction in a higher number of fractions is more effective in terms of radiobiological effects and reduced toxicity, but this study shows that 2 fractions of HDR are comparable with 3 fractions of HDR. This study also shows that BED values calculated using the LQ model are quite useful for comparing different regimens, but a longer followup is required to make a decisive conclusion. Hence in low socio-economic strata patients who discontinue treatment due to economic reasons, a two-fraction regimen of HDR brachytherapy is equally effective.

\section{References}

1. Nandakumar A, Ramnath T, Chaturvedi M. The magnitude of cancer cervix in India. Indian J Med Res 2009; 130: 219-221.

2. Murthy NS, Chaudhry K, Saxena S. Trends in cervical cancer incidence - Indian scenario. Eur J Cancer Prev 2005; 14: 513-518.

3. Juneja A, Sehgal A, Sharma $S$ et al. Cervical cancer screening in India: strategies revisited. Indian J Med Sci 2007; 61: 34-47.

4. Wahi PN, Mali S, Luthra U. Factors influencing cancer of the uterine cervix in North India. Cancer 1969; 23: 1221-1226.

5. Nag S, Erickson B, Thomadsen B et al. The American Brachytherapy Society Recommendations for high dose rate brachytherapy for carcinoma of cervix. Int J Radiat Oncol Biol Phys 2000; 48: 201-211.

6. ICRU Report No 38. Dose and volume specification for reporting intracavitary therapy in gynaecology. ICRU, Bethesda 1985.

7. Jain VS, Sarje MB, Singh KK et al. High-dose-rate-intracavitary brachytherapy applications and the difference in the bladder and rectum doses: a study from rural centre of Maharashatra, India. J Cancer Res Ther 2007; 3: 116-120.

8. Raghukumar P, Raghu K, Saju B et al. Interobserver variation in rectal and bladder doses in the orthogonal films based on planning of treatment of cancer uterine cervix. J Med Phys 2008; 33: 154-157.

9. Mandal A, Asthana AK, Aggarwal LM. Clinical significance of cummulative biological effective dose and overall treatment time in the treatment of carcinoma cervix. J Med Phys 2007; 32: 68-72.

10. Petereit DG, Sarkaria JN, Potter DM et al. High dose rate versus low dose rate brachytherapy in the treatment of cervical cancer: analysis of tumour recurrence - The University of Wisconsin experience. Int J Radiat Oncol Biol Phys 1999; 45: 1267-1274.

11. Ferrigno R, dos Santos Novaes PE, Pellizzon AC et al. Highdose-rate brachytherapy in the treatment of uterine cervix cancer. Analysis of dose effectiveness and late complications. Int J Radiat Oncol Biol Phys 2001; 50: 1123-1135.

12. Busch M, Meden H, Meibodi F et al. Long term results of definitive radiotherapy for cervical carcinoma using four applications of high dose rate afterloading. Cancer 1999; 86: 1520-1527.

13. Roman TN, Souhami L, Freeman CR et al. High dose rate afterloading intracavitary therapy in carcinoma of cervix. Int J Radiat Oncol Biol Phys 1991; 20: 921-926.

14. Patel FD, Sharma SC, Negi PS et al. Low dose rate vs. high dose rate brachytherapy in the treatment of carcinoma of the uterine cervix: a clinical trail. Int J Radiat Oncol Biol Phys 1994; 28: 335-341.

15. Toita T, Kakinohana Y, Ogawa K et al. Combination external beam radiotherapy and high dose rate intracavitary brachytherapy for uterine cervix: analysis of dose and fractionation schedule. Int J Radiat Oncol Biol Phys 2003; 56: 1344-1353.

16. Wong FC, Tung SY, Leunh TW et al. Treatment results of high dose remote afterloading brachytherapy for cervical cancer and retrospective comparison of two regimes. Int J Radiat Oncol Biol Phys 2003; 55: 1254-1264.

17. Liversage WE. A general formula for equating potential and acute regimes of radiation. Br J Radiol 1969; 42: 432-440.

18. Hall EJ. Radiation dose-rate: a factor of importance in radiobiology and radiotherapy. Br J Radiol 1972; 45: 81-97.

19. Dale RG. The application of linear-quadratic dose effect equation to fractionated and protracted radiotherapy. $\mathrm{Br} J$ Radiol 1985; 58: 515-528.

20. Passi K, Maurya AK, Upreti RR et al. Comparison of two different treatment regimens for cancer uterine cervix as practiced in our institute: a retrospective study. J Med Phys 2001; 26: 125-127.

21. Pavy JJ, Denekamp J, Letscert J et al. EORTC late effects working group. Late effects toxicity scoring: The Soma scale. Radiother Oncol 1995; 35: 11-15.

22. Kehwar TS, Passi K, Vashistha R et al. Analysis of combined HDR brachytherapy and external beam radiotherapy in the treatment of carcinoma of cervix. WC 2009 IFMBE Proceedings 2009; 25: 33-36.

23. Passi K, Zakhmi JV, Vashistha R et al. Efficacy of cumulative biological doses at various points of lymphatic trapezoid using microselectron HDR. WC 2009 IFMBE Proceedings 2009; 25: 533534.

24. Passi K, Kehwar TS, Vashistha R et al. High-dose-rate brachytherapy with external beam radiotherapy in the treatment of carcinoma of cervix: dosimetric and radiobiologic analysis. J Radioth Pract 2009; 8: 215.

25. Sood B, Garg M, Avadhani J et al. Predictive value of Linear Quadratic Model in the treatment of cervical cancer using high dose rate brachytherapy. Int J Radiat Oncol Biol Phys 2002; 54: 1377-1387.

26. Han I, Ezzell G, Orton C. HDR Brachytherapy for cancer of the cervix. Veenendaal, the Netherlands: Nucletron BV; 1996 Nucleotron Activity Report No. 8 1996: 22-27.

27. Patel FD, Bhavana R, Indranil $M$ et al. High dose rate brachytherapy in uterine cervical carcinoma. Int J Radiat Oncol Biol Phys 2005; 62: 125-130.

28. Souhami L, Corns R, Duclos M et al. Long-term results of HDR in cancer cervix using a small number of fractions. Gynecol Oncol 2005; 97: 508-513. 